\title{
Comparative Dissolution Studies of Albendazole Oral Suspensions for Veterinary Use
}

\author{
Noelia L. Gonzalez Vidal ${ }^{1, *}$, Walter J. Starkloff', Silvia Bentancor ${ }^{2}$, \\ Silvina Castro ${ }^{3}$, Gonzalo Suarez ${ }^{2}$, and Santiago D. Palma ${ }^{3}$ \\ ${ }^{1}$ Cátedra Control de Calidad de Medicamentos, Departamento de Biología, \\ Bioquímica y Farmacia, Universidad Nacional del Sur, San Juan 670, B8000ICN Bahía Blanca, Argentina \\ ${ }^{2}$ Laboratorio Farmacología, Facultad Veterinaria, Universidad de la República, Alberto Las Places 1550, 11600, \\ Montevideo, Uruguay \\ ${ }^{3}$ UNITEFA-CONICET, Departamento de Farmacia, Facultad de Ciencias Químicas, Universidad Nacional de Córdoba, \\ Ciudad Universitaria, X5000HUA, Córdoba, Argentina
}

\begin{abstract}
Helminth infections are a major health problem mainly in developing countries, and the evidence of emerging resistance to all the major antihelminthics has been recognized in the field of animal husbandry. Albendazole (ABZ) is the most effective broad-spectrum antihelminthic agent. The biggest problem with this drug is its low aqueous solubility, which leads to an erratic availability and great intraindividual variation in the levels reached in different tissues. As with other poorly soluble compounds, the dissolution rate is likely to be contingent on the formulation and may lead to differences in performance among different products available in the market.

In previous studies, drastic pharmacokinetic differences were observed among ABZ formulations (oral suspensions) in the Uruguayan market. These samples were evaluated in vitro in terms of ABZ content and dissolution profiles, as well as the effect of increasing agitation speeds on the dissolution rate. The in vitro dissolution rate of formulation $A$ was seriously affected by the increase in agitation speed, and in the case of formulation B, it did not fulfill both essential quality requirements (drug content and dissolution profile). Further studies may be carried out to correlate the suspension quality with its therapeutic effectiveness.
\end{abstract}

KEYWORDS: Albendazole, suspension, veterinary, dissolution.

\section{INTRODUCTION}

$\mathrm{H}$ elminth infections are a major health problem mainly in developing countries and cause worldwide productivity losses in livestock (1). The economic importance of helminthic infections and the evidence of emerging resistance to all the major antihelminthics in livestock nematodes have been recognized in the field of animal husbandry (2). It is probably for this reason that the most important advances in the chemotherapy of helminthiasis have come from the animal health area (3).

Helminth parasites that have intra- and extraintestinal phases are able to infect humans as well as animals. The treatment for intestinal helminthiasis is usually carried out with benzimidazole drugs, which have a wide spectrum of activity. Albendazole (ABZ), methyl [5-(propylthio)I-H-benzimidazol-2yl]carbamate, is undoubtedly the most effective broad-spectrum antihelminthic agent (4). The biggest problem of benzimidazoles is their low and erratic availability as a result of low aqueous solubility, which leads to great intraindividual variations in the levels reached in different tissues in animals as well as in man.

${ }^{*}$ Corresponding author.
Furthermore, the lack of water solubility reduces flexibility for drug formulation and administration (5).

$A B Z$ is a weak base, so its solubility is higher at low $\mathrm{pH}$ than at neutral $\mathrm{pH}$. The $\mathrm{p} K_{\mathrm{a} 1}$ and $\mathrm{p} K_{\mathrm{a} 2}$ values are 2.68 and 11.83 , respectively. At pH 1.2, the solubility is $900 \mu \mathrm{g} / \mathrm{mL}$, but it is less than $1 \mu \mathrm{g} / \mathrm{mL}$ at $\mathrm{pH}$ values above 5 (6). ABZ is a Class 2 drug in the Biopharmaceutics Classification System (7). This type of drug is absorbed incompletely if not solubilized in the $\mathrm{Gl}$ tract.

Several oral ABZ formulations for use in sheep are available worldwide in the veterinary pharmaceutical market. Some drastic pharmacokinetic differences were observed among commercial ABZ formulations $(8,9)$. Reduced antihelminthic efficacy was associated with $A B Z$ formulations that had the lowest systemic drug exposure (9). The drug content of this oral suspension is critical from a quality control point of view. Suspensions can be considered analogous to the disintegrated form of tablets and capsules, so it is logical to extend the requirement of in vitro dissolution as a limiting step to absorption and bioavailability, especially for drugs with low solubility such as ABZ (10-12). As with other poorly soluble compounds, the dissolution rate is likely to be contingent on the formulation and may lead to differences in performance among different products available in the market (13). The appropriate general conditions 
for performing dissolution profiles of suspensions were established in a previous work (14).

The aim of this work was to evaluate the drug content and dissolution behavior of three commercial ABZ oral suspensions for veterinary use available in the Uruguayan market and the effect of different agitation speeds on the dissolution rate.

\section{MATERIALS AND METHODS \\ Reagents and Samples}

Analytical grade monobasic sodium phosphate and HPLC grade methanol and water were used for chromatographic determinations (J. T. Baker, USA). Analytical grade hydrochloric acid (J. T. Baker, USA) and distilled water were used for dissolution medium preparation. $A B Z$ reference standard was kindly provided by a local laboratory.

Three commercial ABZ oral suspensions manufactured by different pharmaceutical companies were purchased from veterinary shops in Uruguay. They all contained $3.8 \mathrm{~g} \mathrm{ABZ} / 100 \mathrm{~mL}$. All tests were performed within product expiration dates, which were similar among formulations.

\section{Assay}

The drug content was determined using HPLC methodology (15). Reversed-phase HPLC was performed on a system consisting of a quaternary gradient pump (Spectra System P4000), a vacuum membrane degasser (Spectra System SCM1000), a Rheodyne injector (model 9125) with a 20- $\mu \mathrm{L}$ loop, a UV-vis detector (Spectra System UV2000) set at $308 \mathrm{~nm}$, and a chromatography workstation (ChromQuest).

Mobile phase consisted of a mixture of methanol and a $13.75 \mathrm{~g} / \mathrm{L}$ solution of monobasic sodium phosphate (60:40, v/v). Fresh mobile phase was prepared daily, filtered through a 47-mm nylon membrane filter $(0.45-\mu \mathrm{m}$ pore size, $\mu$ clar, Argentina), and vacuum-degassed before use. Separation was performed at room temperature on a Hypersil (Waters) C18 reversed-phase column, 10- $\mu \mathrm{m}$ particle size, $250 \times 4.6 \mathrm{~mm}$ i.d. The column was equilibrated for at least $45 \mathrm{~min}$ with mobile phase flowing through the chromatographic system before starting the assay. All analyses were performed under isocratic conditions at a $2.0 \mathrm{~mL} / \mathrm{min}$ flow rate.

Standard solutions were prepared on weight basis using acidified methanol (methanol/hydrocholoric acid, 99:1 v/v) as diluent and sonicated for $10 \mathrm{~min}$ at room temperature. This stock solution was suitably diluted with mobile phase to obtain a standard solution having a known concentration of about $100 \mu \mathrm{g} / \mathrm{mL}$. An appropriate volume was filtered through a 25-mm nylon membrane disposable filter (0.45- $\mu \mathrm{m}$ pore size, $\mu \mathrm{clar}$, Argentina). They were injected in triplicate (RSD $<2.0 \%$ ), and the results averaged.

Sample solutions were also prepared on a weight basis using a volume of suspension equivalent to $100 \mathrm{mg}$ of
ABZ from each bottle after mixing with a standardized manual procedure, dissolved in acidified methanol, and then treated as the standard solutions. All solutions were used on the day prepared and injected in triplicate.

\section{Dissolution Study}

Since "Albendazole for oral suspension" has no compendial dissolution method, the USP albendazole tablets test was adjusted to serve this purpose (15).

A suspension sample ( $5 \mathrm{~mL}=190 \mathrm{mg} \mathrm{ABZ}$ ) was taken on a weight basis using a suitable syringe-cannula system and transferred quantitatively to the dissolution vessel midway between the surface of the dissolution medium and the top of the rotating blade. Prior to sampling, the reconstituted suspension was mixed by manual agitation (20 upendings in $90 \mathrm{sec}$ ). To calculate the exact weight of suspension added to the vessel, the syringe and cannula were weighed at three stages: empty, filled with the suspension, and after the sample was expelled into the dissolution vessel. The specific gravity of each formulation was determined to express the percentage of drug dissolved in the sampled volume.

Dissolution testing was carried out on a suitably calibrated USP Apparatus 2 (Erweka DT60) at $25 \pm 1 \mathrm{rpm}$, under sink conditions in $900 \mathrm{~mL}$ of $0.1 \mathrm{~N}$ hydrochloric acid solution at $37 \pm 0.5^{\circ} \mathrm{C}$ for each test $(n=12)$. This dissolution medium was used because of the higher solubility of $A B Z$ at acidic $\mathrm{pH}$ compared with neutral or basic media. Samples (10 mL) were withdrawn at 2, 5, 10, 20, 30, 60, and 90 min, with replacement of the same volume of fresh medium after each withdrawal, and filtered through a $25-\mathrm{mm}$ nylon membrane disposable filter $(0.45-\mu \mathrm{m}$ pore size, $\mu$ clar, Argentina). Samples were suitably diluted with dissolution medium and spectrophotometrically analyzed at the $A B Z$ $\lambda_{\max }$ of $310 \mathrm{~nm}$. The concentration in each sample was calculated from an ABZ standard calibration curve. Results were averaged, and cumulative drug release percentages were calculated for dissolution profile estimation. Another test was carried out under the same conditions but using 50 rpm as agitation speed, to evaluate the effect of this parameter in the dissolution rate of the assayed formulations.

Dissolution profiles were compared using the $f_{2}$ similarity factor (16) and ANOVA analysis of Dissolution Efficiency (DE) values. DE is defined as the area under the dissolution curve between two time points (measured using the trapezoidal rule) and expressed as a percentage of the area of the rectangle described by $100 \%$ dissolution in the same time period (17). In all cases, a value of $p<0.05$ was considered significant.

\section{RESULTS AND DISCUSSION}

All formulations evaluated had suitable organoleptic properties and similar specific gravity values $(1.03 \mathrm{~g} / \mathrm{mL}$ for samples $A$ and $B$, and $1.02 \mathrm{~g} / \mathrm{mL}$ for sample $C$ ).

Sample $A$ and $C$ assay results fulfilled pharmacopeial specifications, with average values of $109.4 \%$ and 
$110.0 \%$ of the labeled amount, respectively. Nevertheless, the lowest result was recorded for sample B $(41.8 \%)$, which is well below the specification lower limit for drug content in $A B Z$ oral suspension. ANOVA was used to establish differences between the assay results. There were no significant differences between sample $A$ and $C$ assay values, but statistically significant differences were detected between these formulations and sample B $(p=0.0002)$.

Values for maximum percentage dissolved were near $100 \%$ for samples A and C, with higher dissolution rates for sample $C$ in the study carried out at the recommended agitation speed for suspension evaluation ( $25 \mathrm{rpm}$ ).

Sample A showed the highest standard deviation values, with results over $10.0 \%$ in almost all cases. Variability was in the range of $0.73-1.80 \%$ for sample $C$ and below $1.0 \%$ for sample B. A high dissolution rate was seen for sample $B$, but with a maximum percentage dissolved at $90 \mathrm{~min}$ of only $39.9 \%$ (Figure 1). Although this value shows an inadequate dissolution performance, it is in agreement with the assay result for this sample.

When the dissolution test was carried out at $50 \mathrm{rpm}$, no differences were seen for either sample $B$ or sample $C$. However, the dissolution performance of sample A was seriously affected, which could be related to the formation of a thick clot in the bottom of the vessel. Maximum percentage dissolved was reduced 60 -fold at $50 \mathrm{rpm}$, and the variability in terms of standard deviation also was significantly reduced (Figure 1).

Dissolution profiles were compared in terms of $\mathrm{DE}$, as is shown in Figure 2. Mean DE results were $74.01 \%$, $38.84 \%$, and $87.76 \%$ at $25 \mathrm{rpm}$, and $20.91 \%, 39.46 \%$, and $91.04 \%$ at $50 \mathrm{rpm}$ for samples A, B, and C, respectively. Variability associated with DE in terms of standard deviation was less than $1.0 \%$ in almost all cases, with only one result greater than $8.0 \%$ (sample A, $25 \mathrm{rpm}$ ). ANOVA was used to compare DE values. Significant differences in $\mathrm{DE}$ results were recorded between formulations at $25 \mathrm{rpm}(p<0.01)$ and at $50 \mathrm{rpm}(p=0.0001)$ and between agitation speeds for sample $A(p<0.01)$, sample $B$ $(p=0.0012)$, and sample $C(p=0.0001)$. Nearly the same results were obtained when the profiles were compared via the $f_{2}$ similarity factor, with the exception of the comparison between agitation speeds for samples $B$ and $C$ (Table 1). Therefore, the difference observed between both agitation speeds is meaningful only in the case of sample A.

\section{CONCLUSIONS}

Formulations $\mathrm{A}$ and $\mathrm{C}$ fulfilled pharmacopeial requirements for drug content and showed acceptable dissolution behavior at an agitation speed of $25 \mathrm{rpm}$. Formulation $\mathrm{A}$ dissolution percentages were drastically reduced when the study was carried out at $50 \mathrm{rpm}$, while sample $C$ was almost unaffected. These differences are related to the different compositions (type and amount of excipi-

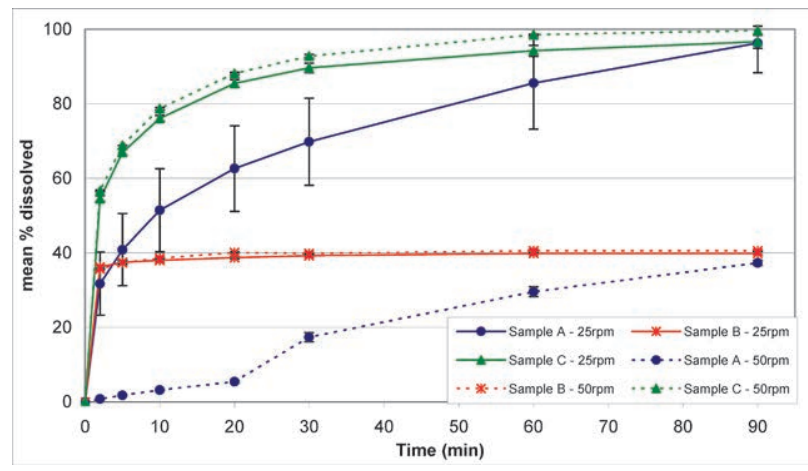

Figure 1. Dissolution profiles of $A B Z$ suspensions at both agitation speeds.

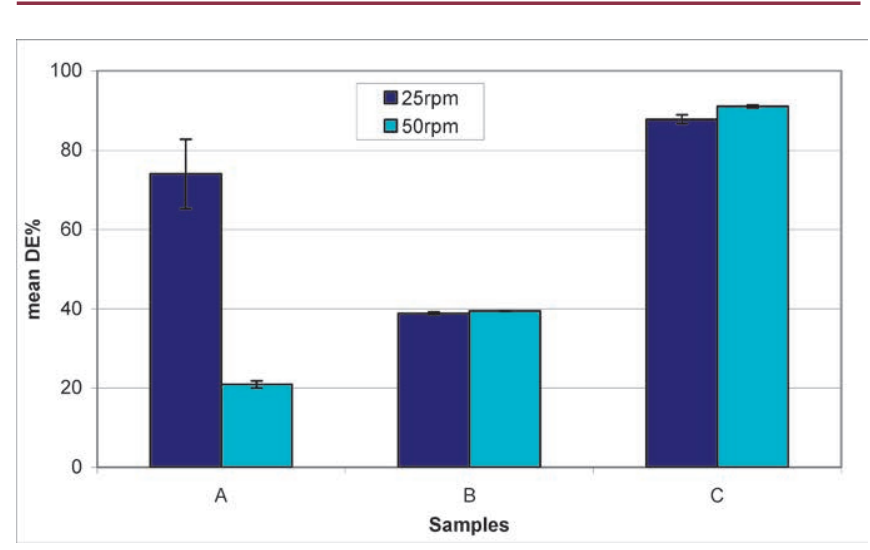

Figure 2. Dissolution Efficiency (DE) results of $A B Z$ suspensions at different agitation speeds.

Table 1. Similarity Factor $\left(f_{2}\right)$ Comparison of Profiles

\begin{tabular}{llccc}
\hline & \multicolumn{3}{c}{$\boldsymbol{f}_{\mathbf{2}}$ Value } \\
\hline \multirow{2}{*}{$25 \mathrm{rpm}$} & Sample A & Sample B & Sample C \\
\cline { 2 - 5 } & Sample A & --- & 21.4 & 31.6 \\
\cline { 2 - 5 } $50 \mathrm{rpm}$ & Sample B & -- & -- & 20.8 \\
\cline { 2 - 5 } & Sample A & --- & 23.8 & 7.2 \\
\cline { 2 - 5 } & Sample B & --- & --- & 19.7 \\
\hline
\end{tabular}

Comparison between agitation speeds ( $25 \mathrm{rpm}$ vs. $50 \mathrm{rpm})$

$\frac{11.4}{\left.{ }^{a} f_{2} \text { values greater than } 50 \text { (i.e., } 50-100\right)}$ ensure sameness or equivalence of
the two profiles.

ents) and manufacturing methods of both formulations. On the other hand, sample B showed the lowest dissolution value, unexpected for a suspension dosage form but in accordance with the drug content result, and was not affected by differences in agitation speeds. This formulation did not fulfill either essential quality requirement, so it would not provide the expected clinical response. Further studies may be carried out to correlate the quality of the suspension with its therapeutic effectiveness. 


\section{CONFLICT OF INTEREST}

The authors declare that there are no conflicts of interest.

\section{ACKNOWLEDGMENTS}

This work was supported by funds from Universidad Nacional del Sur, Argentina (Project number: PGI 24/B139). Walter Starkloff holds a doctoral fellowship of the Universidad Nacional del Sur (UNS), Argentine. The authors would like to thank Asociación de Universidades Grupo Montevideo (AUGM) for the fellowship of Silvia Bentancor.

\section{REFERENCES}

1. Waller, P. J. Global perspectives on nematode parasite control in ruminant livestock: the need to adopt alternatives to chemotherapy, with emphasis on biological control. Anim. Health Res. Rev. 2003, 4 (1), 35-43.

2. Wolstenholme, A. J.; Fairweather, J.; Prichard, R.; von Samson-Himmelstjerna, G.; Sangster, N. C. Drug resistance in veterinary helminths. Trends Parasitol. 2004, 20 (10), 469-476. DOI: 10.1016/j.pt.2004.07.010.

3. Horton, R. J. Benzimidazoles in a wormy world. Parasitol. Today 1990, 6 (4), 106.

4. Cook, G. C. Use of benzimidazole chemotherapy in human helminthiases: Indications and efficacy. Parasitol. Today 1990, 6 (4), 133-136.

5. Lipinski, C. A.; Lombardo, F.; Dominy, B. W.; Feeny, P. J. Experimental and computational approaches to estimate solubility and permeability in drug discovery and development settings. Adv. Drug Deliv. Rev. 1997, 23 (1-3), 3-25.

6. Kohri, N.; Yamayoshi, Y.; Iseki, K.; Sato, N.; Todo, S.; Miyazaki, K. Effect of Gastric pH on the Bioavailability of Albendazole in Rabbits. Pharm. Pharmacol. Commun. 1998, 4 (5), 267-270. DOI: 10.1111/j.2042-7158.

7. Kasim, N. A.; Whitehouse, M.; Ramachandran, C.; Bermejo, M.; Lennernäs, H.; Hussain, A. S.; Junginger, $H$. E.; Stavchansky, S. A.; Midha, K. K.; Shah, V. P.; Amidon, G. L. Molecular Properties of WHO Essential Drugs and Provisional Biopharmaceutical Classification. Mol. Pharm. 2004, 1 (1), 85-96.
8. Eslami, A.; Rassouli, A.; Meshki, B.; Reza Shams, G. A Bioequivalence Study of an Albendazole Oral Suspension Produced in Iran and a Reference Product in Sheep. Int. J. Appl. Res. Vet. Med. 2006, 4 (2), 109-114.

9. Suarez, G.; Alvarez, L.; Castells, D.; Correa, O.; Fagiolino, P.; Lanusse, C. Comparative drug systemic exposure and clinical efficacy against resistant nematodes in lambs treated with different albendazole formulations. J. Vet. Pharmacol. Ther. 2011, 34 (6), 557-564. DOI: 10.1111/j.1365-2885.2011.01274.x.

10. Nahata, M. C.; Jackson, D. S. Stability of cefadroxil in reconstituted suspension under refrigeration and at room temperature. Am. J. Hosp. Pharm. 1991, 48 (5), 992-993.

11. Boonme, P.; Phadoongsombut, N.; Phoomborplub, P.; Viriyasom, S. Stability of extemporaneous norfloxacin suspension. Drug Dev. Ind. Pharm. 2000, 26 (7), 777-779.

12. Guideline for Submitting Documentation for the Stability of Human Drugs and Biologics; Guidelines for Industry; U.S. Department of Health and Human Services, Food and Drug Administration, U.S. Government Printing Office: Washington, DC, February 1987; p 15.

13. Galia, E.; Horton, J.; Dressman, J. B. Albendazole Generics-A Comparative In Vitro Study. Pharm. Res. 1999, 16 (12), 1871-1875.

14. Gonzalez Vidal, N.; Zubata, P.; Simionato, L.; Pizzorno, M. T. Dissolution stability study of Cefadroxil extemporaneous suspensions. Dissolution Technol. 2008, 15 (3), 29-36.

15. The United States Pharmacopeia and National Formulary USP 30-NF 25, Spanish Ed.; The United States Pharmacopeial Convention, Inc.: Rockville, MD, 2007.

16. Moore, J. W.; Flanner, H. H. Mathematical comparison of dissolution profiles. Pharm. Technol. 1996, 20 (6), 64-75.

17. Khan, K. A. The concept of dissolution efficiency. J. Pharm. Pharmacol. 1975, 27 (1), 48-49. DOI: 10.1111/ j.2042-7158.1975.tb09378.x. 